\title{
Que disent les recensions antérieures de recherches sur les enseignements favorisant les compétences en littératie de la fin du primaire et du secondaire?
}

\author{
NANCY GRANGER \\ Université de Sherbrooke
}

\author{
ANDRE C. MOREAU \\ Université du Québec en Outaouais
}

\section{Résumé}

Cet article dresse un état des lieux sur les interventions en lecture et en écriture (stratégies, processus ou modalités) répertoriées depuis trente ans sur les enseignements favorisant les compétences en littératie de la fin du primaire et du secondaire. Notre analyse montre que certaines stratégies cognitives et métacognitives sont bénéfiques pour tous les élèves, qu'ils éprouvent un trouble d'apprentissage ou non. Pourtant elles seraient encore peu présentes dans les pratiques des enseignants. La discussion et la conclusion proposent des pistes de solutions afin que soient optimisées les capacités à lire, à écrire et à communiquer chez tous les élèves.

\section{Mots-clés}

littératie, compétences en littératie, lire et écrire, interventions, enseignement, apprentissage, enseignement disciplinaire

\section{Abstract}

This article provides an overview of the literacy interventions (strategies, processes or modalities) that have been documented over the last 30 years on literacy skills at the end of elementary and secondary school. Our analysis shows that some cognitive and metacognitive strategies are beneficial for all students who have a learning disability or not; yet they would still hardly be present in teachers' practices. The discussion and conclusion suggest possible solutions to optimize the reading, writing and communication skills of all students.

Key words

literacy, literacy competencies, reading and writing, interventions, teaching, learning, disciplinary teaching 


\section{Introduction}

Cet article vise à rendre compte de l'état de l'avancement des recherches recensées sur les interventions ${ }^{1}$ en enseignement de compétences en littératie auprès d'élèves de fin du primaire et du secondaire. L'enseignement de compétences en littératie mène à prendre en compte la diversité de pratiques langagières du lire-écrire, des contextes socioculturels (Dupont et Grandaty, 2015) et des caractéristiques des apprenants (Delarue-Breton et Bautier, 2015). L'étude de ce phénomène de l'enseignement et de l'apprentissage de compétences en littératie peut être comprise comme un ensemble de pratiques d'intervention situées dans un contexte singulier, la classe, qui favorise le développement en langage écrit et, également, des autres disciplines scolaires. Loin d'être un objet nouveau d'études, l'apprentissage du lire-écrire représente un intérêt certain lorsqu'il est croisé avec les élèves en difficulté ou présentant un trouble d'apprentissage. Nos recherches documentaires font ressortir l'importance des recherches et travaux synthèses d'études sur ce sujet ; ce constat a mené à réaliser, dans un premier temps, une analyse des recensions antérieures et, dans une seconde publication, une analyse des écrits scientifiques sur la littératie disciplinaire. Cette double publication sur l'enseignement des compétences en littératie dans différents contextes scolaires permet de faire ressortir les convergences de pratiques d'intervention du lireécrire en langue, mais aussi en enseignement disciplinaire. Les prochaines sections présentent la problématique, la méthodologie de recensions, les interventions en enseignement de la lecture et celles en enseignement de l'écriture ; la discussion qui termine cette synthèse mène à circonscrire les principales conclusions et les pistes de recherches.

\section{Problématique}

La maitrise des compétences en littératie représente un enjeu sociétal, car elle est une condition fondamentale d'accès au savoir (Conseil canadien sur l'apprentissage [CCA], 2011 ; UNESCO, 2008) et, qui plus est, a des conséquences humaines, sociales et économiques sur les sociétés (Word Literacy Foundation, 2015). À cet égard, la tendance des résultats du Programme international pour le suivi des acquis des élèves (OCDE, 2014; PISA, 2012) montre une diminution significative des niveaux d'habiletés en lecture des jeunes canadiens. Bien que les résultats de l'enquête Pisa de 2015 montrent une stabilité depuis 2012, il demeure légitime de se préoccuper des faibles compétences en lecture des jeunes. Cela s'inscrit d'ailleurs dans les visées des ministères de l'éducation canadiens qui préconisent la nécessité travailler ensemble afin de rehausser les résultats d'apprentissage et d'affecter les ressources pédagogiques nécessaires (moyens, méthodes, stratégies) pour relever les défis de notre société (Brochu, Deussing, Houme et Chuy,

\footnotetext{
${ }^{1}$ Le terme intervention utilisé dans ce texte s'appuie sur la définition de Lenoir (2009) et réfère à l'agir de l'enseignant dans ses rapports aux élèves. Sur le plan empirique, opérationnel et pragmatique, l'intervention pédagogique renvoie à l'action de l'enseignant en interaction vers un apprenant ou un groupe d'élèves et vers les savoirs. L'enseignant met en place des dispositifs et processus médiateurs en tenant compte des finalités diverses des acteurs (parents, directions d'établissement, personnel scolaire) et des composantes qui fondent cette interaction apprenant-savoir-enseignant ; cette définition impose un rapport multiple, objectif et subjectif, sur l'acte d'enseignement et l'acte d'apprendre.
} 
2013). Au Québec, plus de $30 \%$ des élèves demeurent en marge de cette visée au terme de leur scolarité, ce qui n'est pas sans préoccuper le Conseil supérieur de l'éducation (CSE, 2013) qui recommande le rehaussement des compétences en littératie des jeunes adultes. Cela rejoint les recommandations du ministère de l'Éducation, des Loisirs et du Sport (MELS, 2008) qui suggère d'accroître le niveau de préparation des enseignants et de renforcer les mesures de soutien en lecture et en écriture; ces propositions représentent des avenues facilitatrices pour améliorer les compétences en littératie des élèves et ainsi leur offrir de meilleures conditions d'apprendre et de mieux réussir.

À cet égard, l'UNESCO $(2015$, p. 1) suggère de faire du droit à l'éducation une réalité afin d'atteindre tous les élèves, en respectant leurs multiples besoins, capacités et caractéristiques, et en éliminant toutes les formes de discrimination dans l'environnement d'apprentissage. Selon Moreau, Hébert, Lépine et Ruel (2013, p.14), la littératie en milieu scolaire rejoint la visée émancipatrice de l'être humain par laquelle les compétences à lire, à écrire et à communiquer efficacement avec différents supports permettent ainsi à la personne de fonctionner dans le quotidien, de développer son potentiel, d'être en interaction avec les autres et le monde, de communiquer, de penser, d'apprendre, de socialiser et d'être critique, de réaliser ses buts et de devenir citoyen du monde. En contexte d'inclusion scolaire, le défi reste entier. Comment le personnel enseignant peutil répondre aux attentes uniformes de fin de cycle du programme en tenant compte de la diversité des apprenants, dont ceux ayant des besoins particuliers ou en difficulté ? En outre, les enseignants font face à un réel dilemme (Maertens, 2004) : plusieurs d'entre eux disent ne pas être suffisamment formés pour relever ce défi (Rousseau, 2015).

Dans l'intention de mieux connaître les facteurs de réussite pour le développement de compétences en littératie, nous avons réalisé une recension d'écrits portant sur les interventions probantes en lecture et en écriture rapportées dans les méta-analyses récentes ou encore dans les synthèses de connaissances. L'objectif est de produire un état des lieux afin d'en dégager les interventions à encourager, de dégager les modalités qui ont fait leur preuve et de proposer des pistes de formation aux enseignants en formation initiale et continue, en vue de soutenir sinon de rehausser les compétences en littératie des élèves en contexte scolaire inclusif.

\section{Méthodologie}

Dans cette recension, la question posée aux écrits scientifiques se résume ainsi : que disent les recensions antérieures de recherches sur les interventions en lecture et en écriture favorables à la réussite des élèves en difficulté à la fin du primaire et au secondaire?

\section{Le point de départ de la recension}

Pour amorcer notre recherche, nous nous sommes d'abord intéressés aux synthèses, revues de littérature ou méta-analyses disponibles en francophonie sur les interventions en lecture et en écriture. En francophonie, une seule synthèse récente correspond aux termes de cette recherche documentaire, soit celle de Bissonnette, Richard, Gauthier et Bouchard (2010). Ces chercheurs ont produit une méga-analyse rapportant l'effet des différentes stratégies en lecture, en écriture et en mathématique sur l'apprentissage des élèves du primaire. Cette méga-analyse porte essentiellement sur des 
études réalisées entre 1999 et 2007. Dans le but de répondre à notre question de recherche qui porte sur les interventions à la fin du primaire et au secondaire, il s'est avéré nécessaire de questionner les bases de données internationales pour la période après 2005.

Les critères de la recension

Prenant appui sur la recension de Bissonnette et al. (2010, p. 8) qui se sont tournés principalement vers les recherches anglophones à cause de leur plus longue tradition d'études expérimentales, nous avons poursuivi notre recension en interrogeant l'outil EBSCO qui comprend plusieurs banques de données en éducation dont Academic search complete, Education source et ERIC. Différents mots-clés ont permis la recension de recherches parues dans des revues avec comité de lecture et impliquant des élèves de la fin du primaire, du début secondaire ou du secondaire (grade 4 to 8; middle school, secondary level, $K-12$ ). Les articles répertoriés portent sur des résultats probants entre les années 2005 et 2016. Les recherches sur le préscolaire et le $1^{\text {er }}$ cycle du primaire ont été exclues de notre recension, de même que celles sur les «nouveaux apprenants en anglais » ou «english learners » étant donné que leur problématique en lecture et en écriture diffère de celle vécue par les élèves à risque et handicapés ou en difficulté d'adaptation et d'apprentissage (HDAA).

En lecture, une première recherche effectuée avec les mots-clés struggling readers and review of the literature a donné 4 articles sur 39 qui correspondaient aux critères. En variant avec struggling students and review of the literature 2 sur 46 articles rencontraient nos critères dont un texte déjà sélectionné. Les mots-clés reading instruction and student wtih disabisabilities and review of the literature or meta-analysis ont permis de sélectionner 3 articles sur 195 trouvés. En écriture, les mots-clés writing instruction and student with learning disabilities and review of the literature or métaanalysis a permis de sélectionner 4 articles sur 60 pour répondre à la question de recherche. Ainsi, sur les 11 articles sélectionnés, 8 correspondent à des méta-analyses, 2 à des revues de littérature quantitatives et 1 à une revue de littérature qualitative. Le tableau ci-dessous résume cette démarche de recherche documentaire. 
Tableau 1

Mots-clés utilisés pour la recension

\begin{tabular}{lc}
\hline \multicolumn{1}{c}{ Mots-clés en lecture } & Articles correspondants \\
\hline $\begin{array}{l}\text { struggling readers and review of the literature } \\
\text { struggling students and review of the literature } \\
\text { reading instruction and student wtih } \\
\text { disabisabilities and review of the literature or } \\
\text { meta-analysis }\end{array}$ & 2 sur 39 \\
\hdashline$\quad$ Mots-clés en écriture & 3 sur 195 \\
\hline $\begin{array}{l}\text { writing instruction and student with learning } \\
\text { disabilities and review of the literature or } \\
\text { méta-analysis }\end{array}$ & Articles correspondants \\
\hline
\end{tabular}

\section{Les limites de la recension}

Dans cette analyse des recensions antérieures, la diversité des protocoles de recherche constitue une première limite. Parmi les études répertoriées, des protocoles expérimentaux, quasi expérimentaux ou encore des études de cas sont répertoriés pour répondre aux questions de recherche. Chaque étude souligne la diversité des données issues de la recherche et la nécessité de redoubler de vigilance dans le choix des données et dans l'établissement de critères à retenir pour les méta-analyses. Aux types de protocoles utilisés s'ajoutent des articles qui proposent plutôt une revue de la littérature sur les objets d'études sans faire une analyse systématique de ces articles scientifiques. Même si cela concourt à une forme de triangulation des données (Mertens, 2005), il s'avère nécessaire de mentionner que nous sommes conscients de la diversité des écrits qui constituent notre recension.

Une deuxième limite concerne l'abondance des méthodes et des interventions réalisées dans les différentes études. Nous avons créé des rubriques qui rendent compte de recoupements entre les discours et les données analysées de façon à ce que le lecteur puisse percevoir les préoccupations scientifiques des chercheurs à travers le temps et puisse identifier les interventions ou les méthodes qui ont fait leurs preuves et qui figurent dans les recensions depuis plus de dix ans. Afin de rendre compte plus adéquatement de l'évolution du concept de littératie à travers le temps et de pouvoir décrire les nouveaux objets des recherches en littératie, deux textes sont proposés dans deux numéros consécutifs de la revue.

Le premier texte traite des recensions antérieures en littératie qui s'intéresse aux interventions probantes en lecture et en écriture. Le deuxième texte présentera les fondements de la littératie disciplinaire (disciplinary literacy) et leur apport quant aux pratiques d'enseignement de sciences, de mathématiques ou d'univers social : comment cette nouvelle conception de la littératie peut enrichir les pratiques d'enseignement et soutenir le développement de lecteurs et de scripteurs lorsqu'ils adoptent une posture disciplinaire.

La première rubrique de cet article présente des recherches sur divers enseignements ainsi que leur apport sur les habiletés en lecture et sur les stratégies 
cognitives en apprentissage. La deuxième rubrique synthétise, quant à elle, les analyses d'études qui documentent des interventions en écriture (stratégies, processus ou modalités). Une discussion et une conclusion proposent une explication des résultats présentés tout au long de cet article et permettent de poser un regard critique sur les recommandations mises de l'avant dans cette recension des recensions antérieures ou méta-analyses.

\section{Interventions en enseignement de la lecture}

De cette recension émergent deux catégories d'écrits. La première comporte des synthèses de recherches qui traduisent les résultats d'interventions spécifiques sur des apprentissages précis de la compréhension en lecture comme le vocabulaire, la fluidité, la capacité de se rappeler une histoire ou à trouver les idées principales d'un paragraphe, etc. La seconde catégorie présente des résultats de recherches qui concernent les interventions les mieux adaptées pour soutenir la réussite des élèves présentant une difficulté d'apprentissage lorsqu'ils doivent lire dans les différentes disciplines. La section cidessous présente l'analyse distincte de chacune des catégories d'écrits.

\section{Les recherches sur l'enseignement de la lecture et l'apport des stratégies cognitives}

En 2009, Edmonds, Vaughn, Wexler, Reutebuch, Cable et Tackett proposent une synthèse des recherches entre 1994 et 2004 auprès d'élèves de la $6^{\mathrm{e}}$ à la $12^{\mathrm{e}}$ année. Leur recension montre que chez les élèves de la fin du primaire et du début du secondaire, les interventions sur la fluidité et la reconnaissance des mots auraient moins d'impact qu'au primaire. Au secondaire, l'enseignement explicite serait un moyen efficace pour intégrer des stratégies relatives à l'enrichissement du vocabulaire et à la compréhension en lecture, d'une part. D'autre part, l'analyse des études recensées suggère également que l'apprentissage de stratégies cognitives et métacognitives associées à l'enseignement du vocabulaire, à la fluidité ou au décodage aiderait les élèves ayant de la difficulté en lecture. Ainsi, entrainer les élèves à utiliser des stratégies cognitives, comme celles de l'autoquestionnement accompagnées de multiples occasions de s'exercer et d'expérimenter des lectures variées, serait profitable à l'engagement des élèves et leur réinvestissent dans divers contextes.

La méta-analyse de Wansek, Wexler, Vaughn et Ciullo (2010) traite, quant à elle, des élèves de $4^{\mathrm{e}}$ et $5^{\mathrm{e}}$ année du primaire ayant des difficultés en lecture. Ces derniers sont considérés par les auteurs comme ayant des besoins similaires à ceux du début du secondaire. Wansek et al. (2010) ont recensé 24 recherches dont les participants avaient principalement entre 9 et 11 ans et étaient décrits comme de faibles lecteurs sans toutefois avoir un diagnostic précis. Leur analyse montre des effets d'ampleur modérés à grands lorsque les interventions portent sur le vocabulaire et les stratégies de compréhension en lecture. Les auteurs concluent que les interventions qui soutiennent la compréhension en lecture et impliquent des processus cognitifs de plus haut niveau outillent davantage les élèves plus âgés que des interventions ciblées comme l'enseignement isolé du vocabulaire ou de la fluidité. Selon eux, des stratégies de lecture comme se référer à ses connaissances antérieures, se questionner avant, pendant et après la lecture et faire un résumé amélioreraient la compréhension. Ils soulignent les résultats obtenus dans les méta-analyses d'autres chercheurs (Kamil, Borman, Dole, Kral, Salinger, et Torgesen

Language and Literacy

Volume 20, Issue 1, 2018
Page 45 
(2008); Scammacca, Roberts, Vaughn, Edmonds, Wexler, Reutebuch, et Torgesen, J. K. (2007) qui montrent que des interventions portant sur plus d'un aspect de la lecture s'avèrent bénéfiques pour les élèves du début du secondaire. Selon Wansek et al. (2010), il serait pertinent d'étudier davantage les interventions mises de l'avant en mettant à profit des évaluations normatives pour mieux documenter les effets de combinaisons d'interventions sur la compréhension en lecture.

La méta-analyse de Flynn, Zheng et Swanson (2012) traite particulièrement d'interventions en lecture à la fin du primaire et au début du secondaire (upper elementary and middle school). Au total, dix recherches ont été répertoriées depuis 1999. Les résultats obtenus montrent un effet d'ampleur modéré des interventions sur l'identification des mots, le décodage et la compréhension alors qu'un effet d'ampleur plutôt faible est constaté en ce qui concerne les interventions portant sur la fluidité en lecture. Les résultats obtenus se basent sur un nombre restreint d'études et les auteurs précisent que leurs résultats doivent être interprétés avec précaution. Ils soulignent d'ailleurs le peu d'études répertoriées chez les élèves plus âgés et appellent à de plus nombreuses recherches en vue de mieux connaître les interventions pour cette tranche d'âge.

La méta-analyse de Scammacca, Roberts, Vaughn et Stuebing (2015) couvre la période de 1980 à 2011 chez des lecteurs en difficultés entre la $4^{\mathrm{e}}$ et la $12^{\mathrm{e}}$ année, soit de la fin du primaire à la fin du secondaire. Les interventions mesurées visaient l'étude de mots, la fluidité, le vocabulaire, la compréhension ou encore des interventions qui ciblaient différentes composantes en lecture à la fois. Cette recherche s'inscrit à la suite d'une première méta-analyse réalisée par Scammacca, Roberts, Vaughn, Edmonds, Wexler, Reutebuch et Torgesen, (2007) portant sur la période de 1980 à 2004. Cette deuxième phase de leur analyse inclut toutes les études précédentes et celles entre 2005 et 2011. Leur question est a) de connaître l'efficacité des interventions en lecture depuis les 30 dernières années; b) de préciser la variabilité des effets selon le devis de recherche utilisée par les divers chercheurs ; et c) de documenter la différence d'efficacité entre les effets répertoriés dans les études plus anciennes et les plus récentes. Au terme de leurs analyses de 82 recherches, les auteurs concluent d'abord que les interventions en lecture répertoriées sont efficaces tant pour les élèves ayant un trouble d'apprentissage que pour ceux qui éprouvent des difficultés en lecture. Ensuite, ils notent que les devis de recherche développés se sont complexifiés depuis la parution de leur méta-analyse de 2007. Le nombre de variables introduites dans les recherches ayant augmenté, les analyses sont plus complexes à réaliser. En effet, la méta-analyse de 2007 obtient des effets d'ampleur plus élevés que celles de 2015. Cette analyse suggère aussi que l'effet d'ampleur est moins grand lorsqu'il s'agit d'évaluations normatives que lorsque les évaluations sont construites par les chercheurs. Les résultats leur permettent tout de même de conclure que des interventions visant l'enrichissement du vocabulaire et la compréhension de texte peuvent être utiles auprès de tous les élèves de la $4^{\mathrm{e}}$ à $1 \mathrm{a} 12^{\mathrm{e}}$ année, qu'ils aient un trouble d'apprentissage diagnostiqué ou non.

En outre, de 2009 à 2015, trois méta-analyses et une recension antérieure de recherche documentent une variété de recherches qui portent sur des enseignements (interventions, stratégies, dispositifs ou méthodes) qui aident à mieux comprendre les retombées sur les composantes de l'apprentissage de la lecture. Pour l'ensemble de ces recensions antérieures synthétisées dans cette partie de texte, retenons que

\begin{tabular}{lll}
\hline Language and Literacy & Volume 20, Issue 1, 2018 & Page 46
\end{tabular}


l'enseignement des premières composantes en lecture ( $p$. ex. décodage, fluidité) est insuffisant en soi et doit être combiné à l'enseignement de stratégies de lecture de haut niveau. En ce sens, l'apport de l'enseignement explicite de stratégies cognitives sur le traitement des informations est clairement démontré. Les interventions documentées ont des effets positifs sur l'apprentissage en lecture tant pour les élèves typiques de la classe ordinaire que ceux ayant des difficultés ou ayant un trouble d'apprentissage. Aussi, considérant que les interventions au primaire et au secondaire sont relativement les mêmes, une continuité de celles-ci, d'un ordre d'enseignement à un autre est souhaitée. En revanche, pour mieux apprécier l'effet d'ampleur de ces interventions, des recherches mettant à profit des outils normatifs d'évaluation des apprentissages en lecture, intégrant diverses composantes simples et complexes ainsi que des stratégies cognitives, doivent être réalisées en ce sens.

Dans un souci de continuité, la prochaine section synthétise les résultats de recensions antérieures qui analysent les recherches sur l'enseignement et l'apprentissage disciplinaire et la place de la lecture et des stratégies cognitives dans ces contextes d'enseignement.

\section{Les recherches sur l'apprentissage et l'enseignement disciplinaire : la place de la lecture}

Dans leur méta-analyse, Kaldenderg, Watt et Therrien (2015) se sont intéressés aux difficultés en lecture en classe de sciences entre la $5^{\mathrm{e}}$ et la $12^{\mathrm{e}}$ année. Au total 12 articles ont été recensés entre 1980 et 2012 portant sur les difficultés éprouvées par les élèves en lien avec la lecture en général, puis les interventions qui s'avèrent utiles pour l'apprentissage par les textes informatifs utilisés en sciences. Ces auteurs soulignent que les élèves ayant un trouble d'apprentissage ont souvent des lacunes en lecture. Lorsque les habiletés de base ne sont pas maitrisées, ces élèves performent sous la moyenne et continuent à prendre du retard par rapport aux autres élèves. Kaldenberg et al. (2015) sont d'avis qu'il est essentiel pour les enseignants des différentes disciplines comme les sciences, les mathématiques ou l'univers social de soutenir ces élèves en difficulté en lecture afin qu'ils s'approprient le contenu disciplinaire et qu'ils puissent tisser des liens entre les stratégies en lecture et les contenus disciplinaires. Pour ce faire, les auteurs se sont questionnés à savoir a) quelles sont les interventions les plus efficaces pour améliorer la compréhension en lecture de textes en classe de sciences ?; b) et quelles sont les variables qui ont un impact sur les interventions en compréhension de lecture en classe de sciences ? En réponse à leur première question, les analyses d'études primaires montrent que pour accroître la compréhension en lecture, l'enseignement du vocabulaire doit se faire en concomitance avec l'organisation graphique. L'utilisation de l'enseignement direct ou le soutien des élèves avec des trucs mnémotechniques seraient aussi pertinents, surtout s'ils sont combinés avec des stratégies métacognitives qui valorisent le questionnement de haut niveau comme se questionner sur les relations de causalité et sur les inférences. Kaldenberg et al. (2015) suggèrent de poursuivre les recherches sur les modalités d'interventions qui combinent l'enseignement de stratégies de lecture et de processus cognitifs pour mieux soutenir les élèves en difficulté, et ce, dans les différentes disciplines.

Pour leur part, McKenna, Shin et Ciullo (2015) ont réalisé une synthèse de 11 études ayant eu lieu entre 2000 et 2013 qui portent sur l'enseignement de la lecture et des 
mathématiques aux élèves ayant un trouble d'apprentissage au primaire et au secondaire. Les auteurs s'intéressaient aux résultats et recommandations formulées lors de la période d'implantation de réformes en éducation. Ces derniers cherchaient à dresser le portrait de

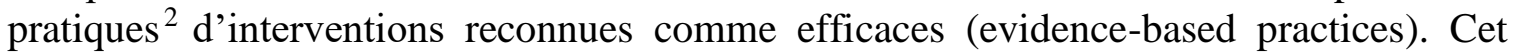
intérêt est lié aux résultats obtenus par Swanson (2008) qui avait étudié l'état des pratiques d'intervention probantes entre les années 1980 à 2005. Ces résultats montraient qu'il y avait peu de soutien en compréhension de texte offert en dépit des recommandations, et que les enseignants appliquaient les stratégies de lecture de façon plus ou moins adéquate. Toutefois, lorsque ces derniers enseignaient le vocabulaire et la fluidité, des résultats encourageants étaient relevés. Enfin, McKenna et al. (2015) concluent que peu de stratégies de compréhension sont enseignées dans ces milieux malgré le nombre élevé d'élèves présentant un trouble d'apprentissage. La majorité des interventions documentées concernaient le groupe classe alors que les interventions individualisées ou en petits groupes étaient peu répertoriées.

Pour faire suite à cette recension d'études primaires et pour mieux comprendre l'évolution de l'implantation de pratiques d'intervention reconnues comme efficaces, McKenna et al. (2015) ont cherché à savoir a) quels sont les domaines et les ordres d'enseignement ciblés par les études primaires auprès d'élèves présentant un trouble d'apprentissage depuis 2000 ?; b) et dans quelle mesure ces recherches recensées documentent-elles les interventions de soutien telles que l'enseignement des stratégies de compréhension ou l'enseignement explicite intégré à un enseignement typique pour les élèves présentant un trouble d'apprentissage ? L'analyse de ces études primaires suggère qu'un écart important existe entre les recommandations en vigueur dans les recherches et les pratiques d'intervention observées dans ces milieux. Les auteurs concluent qu'il y a une plus grande occurrence de l'enseignement de stratégies de compréhension au cycle primaire qu'au secondaire ; dans ces derniers milieux, les pratiques d'intervention visant l'acquisition de stratégies de compréhension sont peu présentes ou même absentes. McKenna et al. (2015) concluent que les recherches futures devraient étudier l'utilisation de stratégies pédagogiques efficaces au sein du curriculum, que ce soit en mathématiques ou dans d'autres disciplines, tant au primaire qu'au secondaire.

Sachant que la lecture à voix haute est un accommodement souvent offert à l'école pour permettre aux élèves présentant un trouble d'apprentissage de mieux comprendre ce qu'ils lisent, Hongli (2014) a réalisé une méta-analyse afin de vérifier si ce traitement est bénéfique uniquement aux élèves ayant un trouble d'apprentissage ou si son usage est favorisé auprès des élèves ayant ou non un trouble d'apprentissage. De même, elle se demandait si des facteurs particuliers avaient une influence sur la compréhension des élèves. Enfin, la chercheuse a comparé les effets obtenus en français et en mathématiques. Les résultats de la méta-analyse de 23 recherches sélectionnées montrent que la lecture à voix haute a un effet bénéfique pour tous les élèves, typique et atypique ; ce résultat montre un effet d'ampleur plus important chez ceux ayant un trouble

\footnotetext{
${ }^{2}$ Le terme pratique désigne « une manière habituelle d'agir » propre à une personne ou un groupe - actions privilégiées - observée dans un ou des milieux d'enseignement; l'usage du qualificatif pédagogique précise qu'il s'agit de l'exercice de la profession enseignante (agir enseignant) (inspirée de Legendre, 2005, p.1065).
} 
d'apprentissage. Parmi les facteurs qui influencent cet accommodement, Hongli note que l'effet serait moins convaincant lorsque l'extrait est lu par un ordinateur, alors qu'un effet positif de lecture par un humain est démontré. Bien que les résultats des analyses montrent un effet significatif plus grand en lecture qu'en mathématiques, l'auteure fait l'hypothèse que les élèves ont possiblement plus d'opportunités de démontrer leur compréhension en mathématiques comparativement à l'évaluation en lecture où seul le texte est proposé comme médium. Enfin, cette méta-analyse ouvre une fenêtre sur une modalité d'enseignement qui semble convenir à plusieurs élèves alors qu'elle est généralement considérée comme une mesure exceptionnelle offerte aux élèves ayant un trouble d'apprentissage.

Donner des consignes qui structurent l'apprentissage, offrir des stratégies qui permettent l'acquisition de connaissances et soutenir les élèves en difficulté ou ayant un trouble d'apprentissage par la lecture à voix haute des consignes sont des moyens recommandés pour soutenir les apprentissages et pour aider les élèves à réussir. Les auteurs de ces recensions antérieures déplorent le faible usage de ces pratiques d'interventions observées dans les milieux et suggèrent qu'elles soient davantage intégrées au sein de la classe.

En somme, les études recensées dans cette section rappellent l'importance d'un enseignement qui intègre des stratégies cognitives branchées sur les besoins des élèves, qu'ils soient en difficulté ou non. Améliorer la compréhension en lecture peut permettre de mieux structurer sa pensée et de faire des liens. En contexte scolaire, les activités d'écriture sont souvent liées à la lecture. La prochaine section présente les interventions qui ont fait leur preuve en enseignement de l'écriture.

\section{Interventions en enseignement de l'écriture}

Écrire à l'école revêt différentes formes. Les élèves ont parfois à répondre à des questions qui nécessitent des réponses courtes et, à d'autres occasions, ils ont à composer un texte avec des informations qui proviennent de sources multiples et qu'ils devront structurer en un tout intelligible selon des genres et des finalités de productions textuelles diversifiées (Bautier et Rayou, 2013) : il s'agit des tâches les plus complexes à réaliser. Cette dernière partie de la recension porte sur les interventions en enseignement de l'écriture qui ont fait leur preuve et qui permettent de répondre aux exigences scolaires tout en développant la capacité des élèves à s'autoréguler lorsqu'ils doivent rédiger des textes, et ce, dans les différentes disciplines. Une première section synthétise la recension de Graham et Harris publiée en 2009 et une deuxième section présente l'analyse de deux recensions des écrits et d'une méta-analyse publiées entre 2012 et 2016.

\section{La recension de Graham et Harris en 2009}

Graham et Harris (2009) proposent une synthèse de recherches recensées, des 30 dernières années, qu'ils ont menée pour documenter des stratégies d'enseignement afin de soutenir les élèves en difficulté ou ceux ayant un trouble d'apprentissage. Ces travaux ont mené à développer le modèle self-regulated strategy development (SRSD) ${ }^{3}$ basé sur la

\footnotetext{
${ }^{3}$ Les six étapes du self-regulated strategy development (SRSD) sont: 1) développer ou activer les connaissances antérieures ; 2) discuter des stratégies à apprendre (Quoi ? Quand ? Comment ?) ; 3) modeler
} 
planification, la révision et l'autorégulation du processus d'écriture dans le but de soutenir les enseignants à composer avec des élèves qui éprouvaient de la difficulté à organiser leur travail, qui possédaient des connaissances de base en écriture lacunaires ou absentes et qui manifestaient peu de motivation à la tâche. Ces élèves démontraient des attitudes négatives, des perceptions de soi erronées, peu d'effort et un sentiment d'efficacité faible. Au fil des recherches réalisées, Graham et Harris arrivent à une conclusion que d'offrir des occasions d'écriture sur une base régulière à ces élèves en difficultés améliorait substantiellement les compétences scripturales. Ils se sont alors intéressés à la formation des enseignants et aux pratiques d'écriture proposées en classe ordinaire (Cutler et Graham, 2008). Ils ont aussi réalisé des revues de littérature portant sur les pratiques d'intervention efficaces en écriture pour les élèves en difficulté ou présentant un trouble d'apprentissage (Graham et Perin, 2007; Rogers et Graham, 2008). Les résultats obtenus mènent aux conclusions que : a) plus les élèves ont de hauts niveaux d'habiletés en écriture, plus ils performent; b) développer des scripteurs habiles nécessite l'enseignement de stratégies plus complexes lorsque le niveau scolaire augmente ; c) la capacité des élèves à utiliser les stratégies enseignées est un bon indicateur des performances en écriture ; et, enfin, d) l'enseignement de stratégies de rédaction permet aux élèves de s'améliorer significativement.

Graham et Harris (2009) rapportent que de proposer ou d'enseigner des procédures de rédaction aux élèves en difficulté améliorent significativement la qualité de leurs textes. La révision de texte de type «phrase par phrase » ou la révision en fonction du contenu du texte peut faire l'objet de consignes ou procédures leur permettant de réguler leurs écrits. De plus, planifier le texte à voix haute avec les élèves et par les élèves permettrait d'éviter une surcharge cognitive et de se centrer davantage sur la tâche (MacArthur et Graham, 1987). L'enseignement de la structure de la phrase en passant du simple au complexe ou encore en combinant deux phrases simples pour en faire une phrase complexe s'avèrent des stratégies qui ont fait leurs preuves. C'est pourquoi, il est recommandé de ne pas utiliser le modèle SRSD lorsque les difficultés des élèves se situent trop à la base, à savoir qu'ils éprouvent de la difficulté à former les lettres, à épeler, ou à choisir les bons mots. Dans ce cas, il serait préférable de consolider ces habiletés de base en premier lieu et de poursuivre avec le SRSD.

Fait intéressant, ces recherches montrent que les élèves en difficulté ou ayant un trouble d'apprentissage mettent davantage l'accent sur la forme et les aspects plus mécaniques de l'écriture (nombre de mots, la propreté, les fautes d'usage) que sur le fond (idées, descriptions, cohérence textuelle). Il est nécessaire d'enseigner explicitement les stratégies de rédaction incluant les étapes de la planification et de la révision. De même, il est essentiel d'enseigner ces stratégies en lien avec le contenu comme celui des genres de textes pour que la performance de ces scripteurs puisse être à la hauteur des attentes relatives à la tâche.

des stratégies; 4) mémoriser les étapes d'application des stratégies; 5) soutenir les élèves durant l'enseignement réciproque ; 6) et évaluer la performance individuelle pour vérifier ce que les élèves ont retenu des stratégies enseignées. Ces étapes peuvent être itératives et sont appliquées selon les besoins des élèves. 
Enfin, Graham et Harris (2009) soulèvent quelques défis relatifs à l'enseignement de l'écriture. Ces derniers ont observé que près de $40 \%$ des enseignants n'enseignent pas de stratégies d'écriture et ne soutiennent pas les élèves ayant une difficulté en rédaction. Ils ont observé également que les élèves ne passent que 20 à 30 minutes par jour à écrire, ce qui serait insuffisant selon eux. Dans ces milieux observés, les enseignants enseignent davantage la grammaire au lieu d'enseigner les processus d'écriture alors que leurs élèves présentent des difficultés. Ces chercheurs ont observé également que l'enseignement de certaines stratégies est inadéquat; ce qui suggère que le personnel enseignant bénéficierait de perfectionnement. Dans ce sens, les auteurs ont documenté que plusieurs enseignants se sentent peu préparés pour enseigner ces stratégies. Peu d'enseignants autres que ceux spécialisés en langue enseignent l'écriture dans leur discipline. Les chercheurs soulignent que les outils technologiques sont encore peu utilisés en classe pour écrire. De plus, le manque de préparation des enseignants toutes disciplines confondues mérite d'être étudié de même que les caractéristiques des enseignants qui influencent le processus d'écriture.

\section{L'analyse de recensions antérieures, période de 2012 à 2016}

Datchuk et Kubina (2012) ont réalisé une revue de littérature sur les habiletés en écriture d'élèves présentant un trouble de l'apprentissage. Dix-neuf articles ont été recensés pour mieux comprendre a) quelles interventions sont utilisées pour enseigner la rédaction de phrase ?; et b) quel effet ont eu les interventions sur les compétences au regard de la rédaction de la phrase et des composantes du texte ainsi que sur le transfert à d'autres tâches plus complexes ? Les résultats montrent que les élèves présentant un trouble d'apprentissage bénéficient d'interventions visant à améliorer la construction de phrase et qu'ils peuvent les réinvestir dans d'autres tâches. Parmi les interventions positives, les chercheurs rapportent l'utilisation d'une phrase simple et un étayage en soutenant de moins en moins (estompage) les élèves pour qu'ils intègrent des éléments plus complexes à l'intérieur de la phrase. Cette intervention peut débuter à partir d'images ou encore en graduant la longueur des phrases. Certaines études rapportent un enseignement de la phrase complexe en combinant deux ou trois phrases simples à l'aide de connecteurs. Différentes déclinaisons de cet enseignement sont décrites. La première consiste à faire travailler des élèves faibles en dyades, une autre en jumelant un élève fort avec un élève faible et une troisième en demandant à des élèves de suivre une méthode répondant aux adverbes où, quand et comment pour décrire l'action. Toutes ces méthodes d'intervention ont montré des résultats positifs tant chez des élèves ayant un trouble d'apprentissage que chez ceux ayant des difficultés. Les auteurs suggèrent de mettre en place des pratiques d'intervention-évaluation qui assurent un suivi des performances des élèves lors de l'enseignement. Ainsi, prendre une mesure au début de l'intervention pour connaître le niveau de performances des élèves et pour documenter cette performance à la suite d'interventions ou d'une séquence d'enseignement permettrait à l'enseignant de réguler sinon d'ajuster son intervention et, qui plus est, de fournir des rétroactions ciblées aux élèves.

Pour leur part, Gillespie et Graham (2014) ont réalisé une méta-analyse des interventions en écriture destinées aux élèves ayant un trouble d'apprentissage. Ils souhaitaient identifier les interventions positives pour améliorer la réussite de ces élèves 
afin de faire ressortir les processus impliqués dans les tâches scolaires en écriture, et ainsi mieux guider les interventions à mettre en place. Au total, 43 études ont été sélectionnées afin de répondre aux deux questions de recherche : a) les interventions en écriture pour tous les élèves sont-elles efficaces pour ceux présentant un trouble de l'apprentissage ?; b) et quelles interventions spécifiques en écriture sont efficaces pour les élèves présentant un trouble de l'apprentissage ? Les résultats montrent que les interventions en écriture ont été efficaces pour tous les types d'élèves, particulièrement ceux de la $4^{\mathrm{e}}$ année et de la $8^{\mathrm{e}}$ année. Sur les six interventions en écriture documentées, l'enseignement de stratégies, la dictée, l'établissement d'objectifs, et l'enseignement de processus d'écriture ont eu des effets positifs sur la performance des élèves ayant un trouble de l'apprentissage. Seule la planification de l'écriture n'a pas permis de conclure à un effet significatif.

Enfin, plus récemment, la recension de Rouhani, Moulavi Nafchi et Mehdi Ziaee (2016) documente quatre méthodes d'enseignement en écriture pour soutenir les élèves ayant un trouble d'apprentissage. Ces méthodes comprennent les éléments comme la tenue d'un journal, l'écriture argumentative, l'enseignement de stratégies d'apprentissage et le développement d'habiletés propres à l'écriture de phrases. Des 15 recherches répertoriées, 13 portent sur l'intervention développée par Graham et ses collègues soit le self-regulated strategy development (SRSD). Les déficits observés chez les élèves en difficulté montrent que ceux-ci peinent à prendre des notes, à surligner et à composer des phrases explicatives, entre autres. Ils ne savent pas comment catégoriser, structurer les informations en tenant compte des idées principales et secondaires. Cette recension montre à quel point les élèves ne possèdent pas de stratégies pour écrire et pour lire en vue de construire du sens en cours d'apprentissage (writing to learn/reading to learn). Les résultats montrent que la stimulation cognitive inhérente au SRSD associée à des moyens pour stimuler les pratiques d'écriture sont efficaces avec tous les types d'écrits (descriptif, littéraire, argumentatif ou informatif) chez les élèves à la fin du primaire et au début du secondaire. Au terme de leur recension, Rouhani et al. (2016) concluent que les pratiques enseignantes éprouvées visant à soutenir l'appropriation de stratégies d'écriture et d'habiletés cognitives font encore peu partie des pratiques de classe. En ce sens, les auteurs recommandent que d'autres recherches puissent documenter les pratiques d'intervention ou d'enseignement qui permettent de soutenir l'apprentissage de différentes habiletés nécessaires à l'écrit. Ces recherches permettraient de mieux cerner le soutien à offrir aux enseignants pour leur permettre d'intégrer dans leur pratique ces interventions reconnues dans les recherches et ainsi favoriser la réussite chez leurs élèves.

En somme, les synthèses de Graham et Harris (2009) ainsi que les recensions subséquentes qui ont eu lieu entre 2012 et 2016 rappellent l'importance de donner aux élèves des moyens pour réguler leurs écrits. Encourager la production de phrases simples et offrir des activités d'enrichissement qui mènent à la rédaction de phrases complexes, et ce, en respectant le rythme des élèves constituent d'excellents points de départ pour engager ces jeunes scripteurs dans leur tâche de rédaction. L'enseignement de stratégies d'écriture associé au modèle self-regulated strategy development (SRSD) contribue à offrir un enseignement-apprentissage centré sur les besoins des apprenants, à faire le suivi des apprentissages, à développer l'autonomie chez les jeunes scripteurs et à soutenir la motivation face à la tâche. 


\section{La discussion}

L'étude de ces écrits scientifiques a permis de synthétiser les résultats d'analyse de 11 recensions antérieures dont les méta-analyses de recherches récentes, depuis 2009, qui éclairent la question que nous posions au départ à savoir: que nous disent les recherches sur les interventions en lecture et en écriture favorables à la réussite des élèves en difficulté à la fin du primaire et au secondaire ?

Comme premier constat, toutes les recensions antérieures consultées montrent que les stratégies d'enseignement éprouvées ou recommandées valent pour tous les types d'élèves, typiques et atypiques, mais démontrent un effet plus marqué chez ceux qui sont en difficulté ou qui présentent un trouble d'apprentissage (Datchuk et Kubina, 2012; Edmonds et al, 2009; Gillespie et Graham, 2014; Graham et Harris, 2009; Hongli, 2014; Kaldenberg et al., 2015; Rouhani et al., 2016; Scammacca et al., 2015). Plusieurs auteurs soulignent les difficultés rencontrées lorsqu'ils tentent d'analyser des résultats de recherches tantôt relatifs à des évaluations construites par les chercheurs, tantôt à des évaluations normatives (Kaldenberg et al., 2015; Scammacca et al, 2015; Wansek et al., 2010). Cela rend difficile la comparaison entre les études ; il s'avère laborieux de préciser les interventions utiles à l'élève et leur incidence sur l'apprentissage.

Parmi les interventions en lecture, l'enseignement du vocabulaire, qui comprend le décodage, la morphologie et l'enrichissement du vocabulaire permet aux élèves de mieux comprendre les textes qu'ils lisent (Edmonds et al., 2009; Flynn et al., 2012; Kaldenberg et al., 2015).

Les recensions antérieures en enseignement de la lecture montrent une réelle différence entre les élèves du début du primaire et ceux qui terminent cet ordre d'enseignement. Ainsi, les élèves de la $4^{\mathrm{e}}, 5^{\mathrm{e}}$ et $6^{\mathrm{e}}$ année semblent avoir des besoins similaires à ceux du début du secondaire. Peu d'études portent encore aujourd'hui sur les élèves de la fin du secondaire et plusieurs chercheurs soulignent la nécessité d'investiguer de ce côté, d'une part (Gillespie et Graham, 2014; Wansek, 2010). D'autre part, plusieurs chercheurs sont d'avis qu'il faut développer des moyens pour étudier de manière plus rigoureuse les interventions mises de l'avant dans les différents milieux pour en dégager des pratiques d'intervention inspirantes en lecture. En effet, avec l'implantation de modèle comme la Réponse à l'intervention ${ }^{4}$ (RÀI) au sein des écoles, il devient essentiel de documenter les démarches expérimentées et les résultats obtenus (Flynn et al., 2012; Kaldenberg et al., 2015; McKenna et al .2015).

En écriture, les interventions basées sur le self-regulated strategy development (SRSD) semblent favoriser une meilleure appropriation du processus scriptural (Graham

\footnotetext{
${ }^{4}$ Selon le National Center on Response to Intervention (2010, p. 2), Réponse à l'intervention (RÀI) réunit des procédés d'évaluation et d'intervention dans un système axé sur la prévention et constitué de paliers multiples pour maximiser la réussite des élèves et pour réduire les problèmes de comportement. Dans ce modèle, les écoles s'appuient sur des données pour identifier les élèves à risque de difficultés d'apprentissage, pour pister les progrès des élèves, pour mettre en œuvre des interventions démontrées efficaces par la recherche, pour adapter l'intensité et la nature de ces interventions à la réponse de l'élève à l'enseignement dispensé et pour identifier les élèves qui présentent un trouble d'apprentissage ou tout autre trouble.
} 
et Harris, 2009). Ce modèle préconise l'enseignement de stratégies cognitives jumelé à un enseignement explicite visant à rendre les apprenants autonomes et aptes à s'autoréguler dans la réalisation des différentes tâches d'écriture scolaires.

Pris ensemble, les résultats de ces recherches en lecture et en écriture s'accordent sur les mêmes principes soit l'importance d'intervenir auprès des élèves afin de leur enseigner des stratégies pour mieux comprendre ce qu'ils lisent et pour diminuer le risque d'échec scolaire (Flynn et al., 2012; Scammacca et al., 2015).

Au secondaire, les élèves, contrairement aux ordres primaires, sont tenus d'utiliser de nombreux éléments du texte pour acquérir des connaissances et pour démontrer une compréhension des contenus enseignés. Si les habiletés en lecture et en écriture ne sont pas acquises à ce stade en raison entre autres de leur historique d'échecs scolaires, cette condition d'apprentissage peut avoir des conséquences graves sur leur engagement face à l'école, et donc, sur leur persévérance (Dynarski, Clarke, Cobb, Finn, Rumberger et Smink, 2008). La nécessité de recourir à des interventions complexes ou méthodes ayant été éprouvées scientifiquement pour intervenir auprès des élèves à la fin du primaire, avant qu'ils n'atteignent le secondaire, prend alors tout son sens.

\section{Conclusion}

L'analyse des recensions antérieures de recherches sur les enseignements favorisant les compétences en littératie de la fin du primaire et du secondaire montre que l'enseignement de stratégies de lecture associées à stratégies cognitives et métacognitives, aux élèves en difficulté ou avec un trouble d'apprentissage, constitue une voie à emprunter pour qu'ils créent davantage de liens et performent mieux dans les tâches scolaires à réaliser. Les recherches montrent avec éloquence qu'il reste beaucoup de chemin à parcourir pour que l'enseignement de ces stratégies soit pris en compte par le personnel enseignant et fasse partie de leur culture d'enseignement.

En réponse à ce problème, le deuxième article de cette série de deux présente l'apport de la littératie disciplinaire tout en réfléchissant sur un mode de planification et de gestion pédagogique ou didactique propice à cette construction de sens entre le texte lu, son analyse et le réinvestissement dans une forme de production de textes écrits en contexte d'enseignement de différentes disciplines scolaires. Les questions soulevées sont les suivantes: comment articuler l'apprentissage général à l'apprentissage spécifique de façon à générer des stratégies transdisciplinaires de littératie permettant ainsi d'aborder différents types de textes et d'y réagir à l'oral ou à l'écrit. Quels peuvent être les apports des disciplines au développement de stratégies de littératie, lire et écrire ? Comment arrimer les différentes disciplines autour de pratiques d'intervention communes pour tisser des liens entre les savoirs enseignés dans chaque discipline ?

Voilà quelques questions auxquelles chercheurs et praticiens devront réfléchir afin que l'école forme des jeunes capables de lire, d'écrire et de communiquer dans différents contextes en mobilisant les savoirs acquis et les compétences développées au travail et dans les différents contextes d'apprentissage tout au long de la vie. 


\section{Références}

Bautier, E. \& Rayou, P. (2013). La littératie scolaire : exigences et malentendus. Les registres de travail des élèves. Éducation et didactique, 7(2), 29-46.

Bissonnette, S., Richard, M., Gauthier, C., \& Bouchard, C. (2010). Quelles sont les stratégies d'enseignement efficaces favorisant les apprentissages fondamentaux auprès des élèves en difficulté de niveau élémentaire ? Résultats d'une mégaanalyse. Revue de recherche appliquée en apprentissage. 3(1), 1-35.

Brochu, P., Deussing, M.-P., Houme, K. \& Chuy, M. (2013). À la hauteur : Résultats canadiens de l'étude PISA de l'OCDE. Le rendement des jeunes du Canada en mathématiques, en lecture et en sciences. Premiers résultats de 2012 pour les jeunes du Canada âgés de 15 ans. Toronto, On. : Conseil des ministres de l'éducation (Canada). Repéré à http://cmec.ca/Publications/Lists/Publications/Attachments/318/PISA2012_Canad ianReport_fr_Web.pdf

Conseil canadien sur l'apprentissage (2011). Quel est le futur de l'apprentissage au Canada? Ottawa : Conseil canadien sur l'apprentissage. Repéré àwww.ccl-cca.ca.

Conseil supérieur de l'éducation du Québec (2013). Un engagement collectif pour rehausser les compétences en littératie des adultes. Avis à la ministre de l'Éducation, du loisir et du sport et au ministre de l'Enseignement supérieur, de la recherche, de la science et de la technologie : Québec. Repéré à http://www.cse.gouv.qc.ca/fichiers/documents/publications/Avis/50-0482.pdf

Cutler, L., \& Graham, S. (2008). Primary grade writing instruction: A national survey. Journal of Educational Psychology, 100, 907-919.

Datchuk, S. \& Kubina, R.M. (2012). A review of teaching sentence-level writing skills to students with writing difficulties and learning disabilities. Remedial and special education, 34(3), 180-192.

Delarue-Breton, C. \& Bautier, E. (2015). Nouvelle littératie scolaire et inégalités des élèves : une production de significations différenciée. Le français 190(3), 51-60.

Dupont, P. \& Grandaty, M. (2015). La notion de poste de travail didactique et le développement des compétences en littératie d'élèves de cycle 3. Dans Lizanne Lafontaine et Johanne Pharand, Littératie. Vers une maîtrise des compétences dans divers environnements (p.57-86). Québec : Presses de l'Université du Québec.

Dynarski, M., Clarke, L., Cobb, B., Finn, J., Rumberger, R., \& Smink, J. (2008). Dropout Prevention: A Practice Guide. National Center for Education Evaluation and Regional Assistance, Institute of Education Sciences, U.S. Department of Education; Washington. Repéré à: http://ies.ed.gov/ncee/wwc.

Edmonds, M. S., Vaughn, S., Wexler, J., Reutebuch, C. K., Cable, A., \& Tackett, K. K. (2009). A synthesis of reading interventions and effects on reading outcomes for older struggling readers. Review of Educational Research, 79, 262-300.

Flagella-Luby, M.N., Sampson Graner, P., Deshler. D. D., \& Valentino Drew, S. (2012). Building a house on sand: Why disciplinary literacy is not sufficient to replace general strategies for adolescent learners who struggle. Topics in language disorders, 32(1), 69-84.

Flynn, L. J., Zheng, X., \& Swanson, H. (2012). Instructing struggling older readers: A 
selective meta-analysis of intervention research. Learning Disabilities Research and Practice, 27, 21-32.

Gillespie, A. \& Graham. A. (2014). A meta-analysis of writing interventions for students with learning disabilities. Exceptional children, 80(4), 454-473.

Graham, S. \& Harris, K.R. (2009). Almost 30 Years of Writing Research: Making Sense of It All with The Wrath of Khan. Learning disabilities research and practice, 24(2), 58-68.

Graham, S. \& Perin, D. (2007). A meta-analysis of writing instruction for adolescent students. Journal of Educational Psychology, 99, 445-476.

Hongli, L. (2014). The effects of read-aloud accommodations for students with and without disabilities: A meta-analysis. Educational Measurement: Issues and Practice, 33(3), 3-16.

Kaldenberg, E. R., Watt, S.J., \& Therrien, W.J. (2015). Reading Instruction in Science for Students With Learning Disabilities: A Meta-Analysis. Learning Disability Quarterly, 38(3), 160-173.

Kamil, M. L., Borman, G. D., Dole, J., Kral, C. C., Salinger, T., \& Torgesen, J. (2008). Improving adolescent literacy: Effective classroom and intervention practices: A Practice Guide. Washington, DC: National Center for Education Evaluation and Regional Assistance, Institute of Education Sciences, U.S. Department of Education.

Legendre, R. (2005). Dictionnaire actuel de l'éducation (3e édition). Guérin éditeurs: Montréal.

MacArthur, C. \& Graham, S. (1987). Learning disabled students' composing under three methods of text production: Handwriting, word processing, and dictation. Journal of Special Education, 21, 22-42.

Maertens, F. (2004). Évolution des services éducatifs publics du Québec destinés aux élèves ayant des besoins particuliers. Dans N. Rousseau \& S. Bélanger (dir.), La pédagogie de l'inclusion scolaire (pp. 22-40). Sainte-Foy : Presses de l'Université du Québec.

McKenna, J.W., Shin, M., \& Ciullo, S. (2015). Evaluating reading and mathematics instruction for students with learning disabilities: A synthesis of observation research, Learning disability quarterly, 38(4), 195-2017.

Mertens, D.M. (2005). Research and evaluation in education and psychology: integrating diversity with quantitative, qualitative, and mixed methods. Thousand Oaks, CA : Sage Publications.

Ministère de l'Éducation, du Loisir et du Sport (2008). Mieux soutenir le développement de la compétence à écrire. Rapport du comité d'experts sur l'apprentissage de l'écriture.

Moreau, A.C., Hébert, M., Lépine, M., \& Ruel, J. (2013). Le concept de littératie en francophonie : que disent les définitions? Revue Consortium national de recherche sur l'intégration sociale (CNRIS), 4(2), 14-18.

OCDE (2014). Principaux résultats de l'enquête Pisa 2012 : ce que les élèves de 15 ans savent et ce qu'ils peuvent faire avec ce qu'ils savent. Repéré à https://www.oecd.org/pisa/keyfindings/pisa-2012-results-overview-FR.pdf

Rogers, L. \& Graham, S. (2008). A meta-analysis of single subject design writing 
intervention research. Journal of Educational Psychology, 100, 879-906.

Rouhani, Y., Moulavi Nafchi, A., \& Mehdi Ziaee, S. (2016). Applying Different Interventions to Teach Writing to Students with Disabilities: A Review Study. Theory and Practice in Language Studies, 6(4), 733-741.

Rousseau, N. (2015). La pédagogie de l'inclusion: un défi ambitieux et stimulant (3e Éd.). PUQ: Québec.

Scammacca, N., Roberts, G., Vaughn, S., Edmonds, M., Wexler, J., Reutebuch, C. K., Torgesen, J. K. (2007). Reading interventions for adolescent struggling readers: A meta-anal-ysis with implications for practice. Portsmouth, NH: RMC Research Corporation, Center on Instruction.

Scammacca, N.K., Roberts, G., Vaughn, S., \& Stuebing, K. (2015). A Meta-Analysis of Interventions for Struggling Readers in Grades 4-12: 1980-2011. Journal of Learning Disabilities, 48(4) 369-390.

Swanson, E. (2008). Observing reading instruction for students with learning disabilities: A synthesis. Learning Disability Quarterly, 31, 115-133.

UNESCO (2008). Education for all by 2015: We'll we make it? Repéré à: http://en.unesco.org/gem-report/report/2008/education-all-2015-willwe-make-it\#sthash.z6HghGqs.dpbs

UNESCO (2015). Forum mondial sur l'éducation. Repéré à: http://fr.unesco.org/worldeducation-forum-2015/5-key-themes/education-inclusive

Wansek, J. Wexler, J. Vaughn, S., \& Ciullo, S. (2010). Reading interventions for struggling readers in the upper elementary grades: a synthesis of 20 years of research. Reading and writing, 23(8), 889-912.

World Literacy Foundation (2015). The Économic \& Social Cost of Illeteracy. A snapshot of illiteracy in a global context. Final report from the World Literacy Foundation. Repéré à https://worldliteracyfoundation.org/wp-content/uploads/2015/02/WLFFINAL-ECONOMIC-REPORT.pdf 


\section{Biographie des auteures}

Nancy Granger, $\mathrm{Ph}$. D. est professeur adjointe à l'Université de Sherbrooke où elle est coresponsable du Microprogramme de $2^{e}$ cycle en conseillance pédagogique au département de Gestion de l'éducation et de la formation. Elle est chercheuse au sein de l'Équipe de recherche en littératie et inclusion (ÉRLI) où elle a réalisé un postdoctorat. Ses recherches portent sur la formation continue et l'accompagnement des enseignants, l'inclusion scolaire des élèves ayant des besoins particuliers, la littératie ainsi que sur les pratiques d'enseignement et d'apprentissage. Des recherches-actions ont également été réalisées sur le thème de l'organisation des services notamment au regard de la fonction d'enseignant-ressource au secondaire et sur le dispositif de coenseignement. Des articles scientifiques et chapitres de livres ont été publiés sur ces différents thèmes.

André C. Moreau, Ph. D. est professeur agrégé à l'Université du Québec en Outaouais. Ses recherches portent sur la littératie et l'inclusion des élèves ayant des besoins particuliers et sur les pratiques d'enseignement et d'apprentissage. Il est cotitulaire de la Chaire interdisciplinaire de recherche en littératie et inclusion - CISSS de l'Outaouais ainsi que chercheur d'Équipe de recherche en littératie et inclusion (ÉRLI). Des recherches-actions ont également été réalisées sur les thèmes de transitions scolaires et de communautés d'apprentissage professionnelles. Il a publié plusieurs articles scientifiques, livres et rapports de recherche sur ces thèmes. 